\title{
Summary of Power Grid Research in Smart Home Environment
}

$$
\text { Xin Wang }{ }^{1} \text {, Ziyao Zou }{ }^{1} \text {, Yan } \mathrm{Wu}^{1} \text {, Zhijun } \mathrm{Gao}^{2, \mathrm{a},{ }^{*}} \text { and Xiaofu } \mathrm{Ma}^{3}
$$

${ }^{1}$ School of information and control engineering, Shenyang Jianzhu University, Shenyang, China

${ }^{2}$ Graduate School, Shenyang Jianzhu University, Shenyang, China

3Wireless@VT, Virginia Tech,Blacksburg, VA, USA

aarnold0110@sina.com

* Graduate School, Shenyang Jianzhu University, Shenyang, China

Keywords: Smart home; Smart grid network; Real-time pricing; Optimize in power source; Security

\begin{abstract}
Our living and electricity system have been inseparable. Using the smart grid in the daily life is increasing the users' living quality and reducing the cost of electricity. This paper explains how to set the electronic facility according to different real-time pricing, and then it reaches an effect on using the electricity efficiently. In smart home, optimal of power source is an extending of smart grid. Therefore, the article also writes how to reduce the cost of electricity by use optimal of power, in turn we can realizes win-win in intelligent and economic. Nevertheless, there are series of problems in electrified wire netting security; we analyze electrified wire netting security from three aspects. We importantly explain technique how to detect the invading.
\end{abstract}

\section{Introduction.}

Smart grid is the newest energy system .The users of electrical power make response through market price. Requirement response could be realized by real-time pricing. Recently both in domestic and foreign market, there are a lot of literature study real-time pricing, some literature design strategy about using electricity via non-cooperative game, the way reduces peak to average rate of aggregate demand on electrical power. Some literature set real-time pricing based on maximizing the using efficiency. However, all literature did not consider a question that whether the supplier of the electrical power can satisfy users' requirement. So this article will present an approach based on admission control. It is how to accumulate the energy in the low price period and utilize these energies in the high price period to meet the demand of users[1,2,3,4].

Energy problem had been into the smart home and it is also a direction of smart grid network. Optimize in power source is an important factor to keeping the sustainable development of smart home. The data shows that $36.6 \%$ of electricity consumption is living electricity, but the research presents that the living electricity consumption is actually low efficiency and waste a lot. The literature provides a way of power source based on MPC [5,6].

However, the public network provides many entrances because of hostile attack of the public network. The electrified wire netting and its users will face a serious problem.

\section{Development of Real-time Pricing and Best Way to Using the Electricity}

Fundamental Form of Real-time Pricing. In the environment of real-time pricing, real-time pricing is the function of aggregate demand of electrified wire netting. So the slot time is $\mathrm{K}$, users is $\mathrm{N}, \quad l_{k}$ is amount to supply of the slot time, $i \in N, x_{k}^{i}$ means electricity consumption by user $\mathrm{i}$ in slot time. We assume the minimum electricity consumption is $m_{\mathrm{k}}^{\mathrm{i}}$ by user $\mathrm{i}$ at slot time, the electricity consumption by user i satisfied following rule: $x_{k}^{i} \geq m_{k}^{i}$.

Arithmetic of Admission Control. This part presents the design steps that user cannot reach minimum electricity consumption $m_{\mathrm{k}}^{\mathrm{i}}$. Firstly, we can compare the amount of electricity

Consumption $(\mathrm{u})$ which is user get and minimum electricity consumption by user $(\mathrm{v})$, if $\mathrm{v}<\mathrm{u}$, 
then the electricity supplier stops send electricity purchasing message. The users that have not to reach the electricity purchasing condition stop electricity purchasing, the supplier does not need to send a feedback message to ensure. So the arithmetic of admission control 1 is finished. Or choosing the user who gets a biggest gap between actual electricity consumption and minimum demand of electricity consumption to stop electricity purchasing and send the feedback to the users. If user did not get purchasing message, then arithmetic 2 is finished. In the arithmetic 2, users stop the purchasing after receives the feedback message, and then users choose other supplier, otherwise, transform into the first step.

The Assessment about Performance of Arithmetic of Admission Control. We assume the amount of electricity supplied by slot time supplier unsatisfied all of the users' minimum requirement .Even electricity consumption and minimum demand, we can know the relationship between user 1 and user 2 who unsatisfied minimum demand as following.

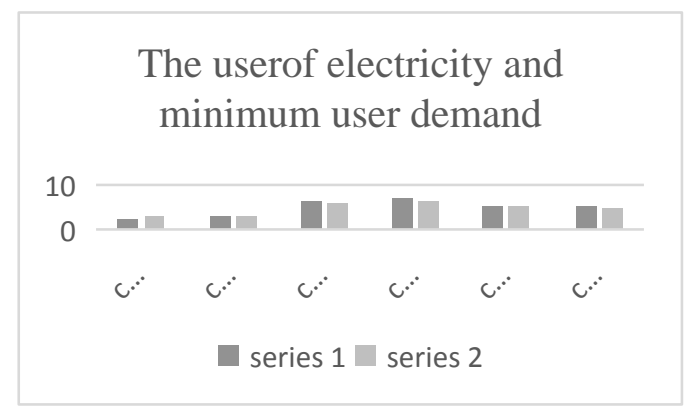

Figure 1. The user of electricity and minimum user demand

According to Fig .2, user2 and user1 get satisfied minimum electricity demand. Thus arithmetic 2 is suitable for living power saving.

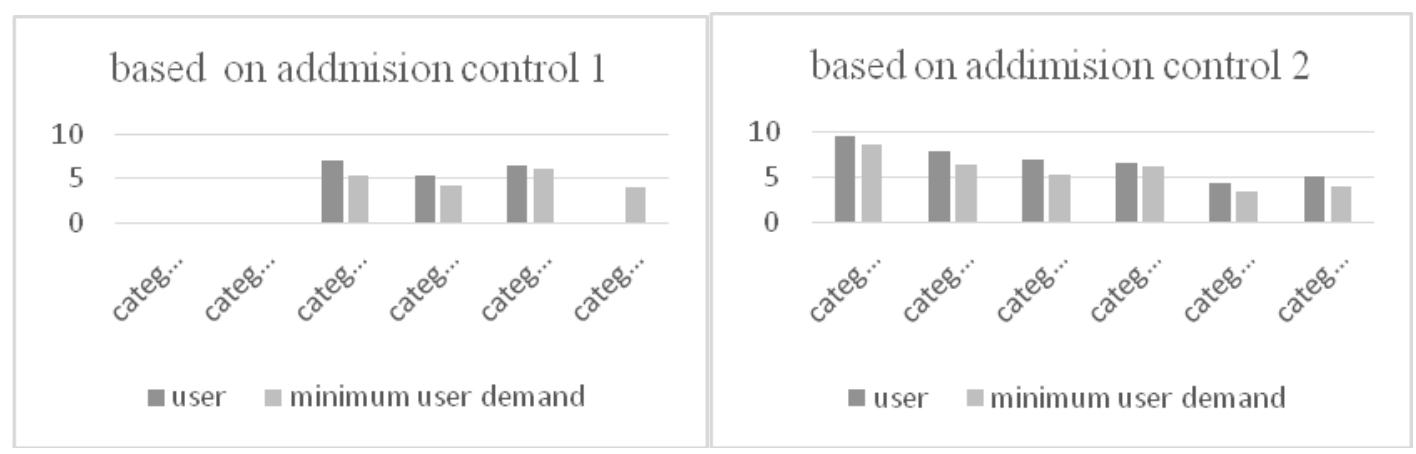

Figure 2. Based on admission control

Adjustment on Optimized Household Electricity. Normally energy is known as source which provides light, heat, electricity and power etc. Now the thing we need to do is make the operation of smart home system with the lowest cost. And we do it by optimize the controlling of electrical equipment, the literature talks about the optimization which based on MPC [7,8,9].

Principle of MPC. MPC is a kind of closed loop optimal control algorithm. The optimization process takes the system state of the current time as the observation. It is solve the optimal problem through move ways. it is fit unsure problem.

Electricity Dispatching Algorithm. Input means the data of living sensor and wearable sensor on time $\mathrm{k}$.Output means the electrical control strategy $\mathrm{q}(k)$. Firstly, temperature of time $\mathrm{k}$ is $x_{m}(k)$, we observe the living environment, update system model $\phi(a, \beta, \delta, g)$ and demand of users' comfort level $\mathrm{R}\{\mathrm{z}, \overline{\mathrm{T}}\}$, we also forecast state information according to state system model

$\hat{x}_{m}(k+\mathrm{n} \mid k)$.we use household equipment $\left.\hat{q}(k)\right|_{k} ^{K-1}$ in the literature. 


$$
\begin{aligned}
& k \min j=\sum_{k}^{K-1} p(k) \hat{q}(k) \\
& x_{i n}(k+1)=\mathrm{ax}_{\mathrm{in}}(k)+\beta \mathrm{x}_{\mathrm{out}}(k)+\delta \hat{\mathrm{q}}(\mathrm{k})+\mathrm{g}, \\
& 0 \leq \hat{q}(k) \leq q_{\mathrm{max}} \\
& \bar{T}-\Delta \leq \mathrm{x}_{\mathrm{in}}(k) \leq \bar{T}+\Delta, \text { if } \mathrm{z}(k)=1
\end{aligned}
$$

$J$ represents the cost of electricity at optimized periods. K we already know; we can solve this problem by linear programming. Then we need to put the value of the optimal starting time be applied to the system, $q(k)=\hat{q}(k)$, at the end let $\mathrm{k}=\mathrm{k}+1$, go back to the step 1 let $\mathrm{k}=\mathrm{K}-1$.

The Assessment that Based on this Algorithm: The literature assesses the effect on economic aspect. Economy is important standards to evaluate the algorithm whether appropriate, and then the algorithm can meet our smart home economy, energy saving, comfort etc. The following chart shows that this scheme can be implemented. The following is the case of electricity under various scenarios.

Table 1 The case of electricity under various scenario

\begin{tabular}{|l|l|l|l|l|}
\hline policy & Scenario1 & Scenario2 & Scenario3 & Scenario4 \\
\hline Rule-based & 3.56 & 4.67 & 3.77 & 3.24 \\
\hline MPC & 3.12 & 3.45 & 2.90 & 2.33 \\
\hline Improvement/\% & $12.4 \%$ & $26.1 \%$ & $23.0 \%$ & $28.1 \%$ \\
\hline
\end{tabular}

\section{Smart Grid Network Security Technology}

IDS. IDS is according to a certain rule or system analysis. Simple to say it is a technology that report unauthorized and abnormal phenomenon. In the process of combining power system and network, the application of intrusion detection technology is strengthened, that is to monitor and audit the program and harmful code. The following graph is basic model for IDS.

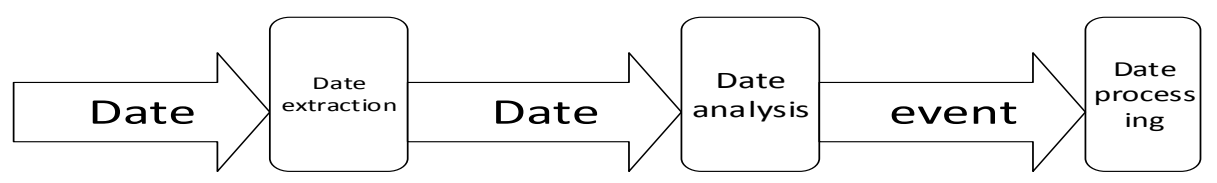

Figure 3. Basic model for IDS

Basic Principles and Classification for IDS. In the IDS, the system is not reliable, if it is not authorized to attempt to access information and tamper with the information. Intrusion detection can identify intruders and intrusion behaviors, and a perfect intrusion detection system has the characteristics of economy, timeliness, security, extendibility, and so on.

IDS Based on Large Data. IDS Based on large data is make it a massive amounts on the breadth and the recording time span growth data through the security audit data and it is based on the significant improvement of the modern storage capacity, network bandwidth and computing performance. We use a large number of intelligent ammeter upload safety design massive data, the way makes management center identify abnormal meter by big data. In order to detect malicious intrusion behavior rapidly from the abnormal behavior of the network, the network security protection level of the information system is expected to be significantly improved.

Anti-Virus Technology (AVT): With the development of electric power system, the level of information technology also keeping develop, the internet which connects with external will affect by external viruses easily. So it is necessary that the electricity companies to install anti-virus software and anti-virus network. 
The Design Content of the Latest Anti-virus Software. With the deployment of new technologies to defend against unknown vulnerabilities, the new technology inspired zero-day attacks and continue to increase the user unknowingly attack. For example, bank password may be captured, and a smart grid system may be paralyzed. Thus the zero-day affects everyone's living quality. In Abroad, the latest research method is through the virtual system and method of designing anti-virus software. Anti-virus system can through virtualization separation remain user application program and the operating system,

\section{New Technology for Data Backup}

Hadoop. It is through a single storage medium to achieve the traditional data backup technology, it is simple and cheap, but the security is low and the extendibility is poor. In recent years, a distributed technology is rising, it can realize the data distributed storage and computing, Hadoop technology as representative of distributed technology; it can make full use of the resource nodes, good safety, expansion and efficiency.

Cloud AC .With an extending of WLAN network building, there are many weaknesses disclosed. In order to change present situation of WLAN, we put the Cloud Computing into the WLAN. The WLAN structure based on Cloud AC is a direction for developing of Current Wireless local area network. The thinking of Cloud Computing has been used in WLAN network; we integrate all the AC resources. Come into being Ac cloud Offer service for outside through the way. And then we can improve the network utilization and reliability, and optimize the network resources.

\section{Summary}

From two aspects of smart home, smart grid is well explained. One is by using electric power more economically (which makes our life comfortable), the other is to protect our security about electric power. We apply electric power communication technology to power grid, improve and develop it scientifically, and solve the problems such as network security and energy optimization on the field of smart home, in order to monitor household goods and appliances as well as collect data. Furthermore, we realize transmission and communication of data by the communication network.

\section{References}

[1] B. Fan, Y. Zeng, “Vulnerability Assessment of Power Communication Network Based on Information Entropy”, Electronics \& Information Technology, 2004, vol. 36, no. 9, pp. 2138-2143.

[2] D.Y. Fang, S.D. Qi and Z.Y. Tang, “An Evil-Twin AP Detection Method Based on RSSI in Smart Home” , Journal of Computers, 2016, vol. 39, no. 1, pp. 1-15.

[3] Xiaofu Ma, Thaddeus Czauski, Taeyoung Yang, and Jeffrey H. Reed, “Demonstration Paper: Wirelessly Sensing Medication Administration: Cyber-Physical Event Detection and Notification Utilizing Multi-Element Chipless RFID” , Proceedings of the Wireless Health, 2014, pp.1-8.

[4] K. Ma, T. Yao and X.P. Guan, "Demand response based on admission control in smart grid” ,Control and Decision, 2015, vol. 30, no. 1, pp. 190-192.

[5] X. Zhang, D. Han, G.H. Cao, “Security authentication protocol for RFID based on PRESNT algorithm” , Communications, 2015, vol. 36, no. 21, pp. 65-74.

[6] W.Y. Gao, “Research of Admission Control in IP Network” , Computer Engineering, 2005, vol.31no. 1, pp.1-2. 
[7] Xiaofu Ma, Haris I. Volos, Xiangwei Zheng, Jeffrey H. Reed, and Tamal Bose, "A Variation-Aware Approach for Task Allocation in Wireless Distributed Computing Systems” , IEEE Global Communications Conference, pp. 5006-5011, 2013.

[8] Y. Xu,Y. H. Lv, "Analysis of the Coexistence of Indoor Broadband Communication Network for Smart House” , Electronics \& Information, 2008, vol. 30, no. 9, pp. 2233-2237.

[9] Xiaofu Ma, Yu Fang, Xingzhen Bai, “A Balanced Energy Consumption Clustering Algorithm for Heterogeneous Energy Wireless Sensor Networks", IEEE International Conference on Wireless Communications, Networking and Information Security, pp.382,386, 2010. 